\title{
Decisive influence of substitution positions in molecular self-assembly
}

\author{
Julia L. Neff, Markus Kittelmann, Ralf Bechstein and Angelika Kühnle*
}

\begin{abstract}
Molecular self-assembly provides a versatile tool for creating functional molecular structures at surfaces. A rational design of molecular structure formation requires not only an in-depth understanding of the subtle balance between intermolecular and molecule-surface interactions, but might also involve considering chemical changes of the molecules, such as deprotonation. Here, we present a systematic investigation of a comparatively simple class of molecules, namely dihydroxybenzoic acid, which, nevertheless, enables creating a rich variety of structures when deposited onto calcite (10.4) held at room temperature. Based on non-contact atomic force microscopy measurements in ultra-high vacuum, our study demonstrates the decisive impact of the positions of the hydroxyl groups on the structure formation. Six isomers of dihydroxybenzoic acid exist which form six different molecular structures on the calcite surface. Surprisingly, only two isomers arrange into stable, ordered structures at submonolayer coverage: 2,5-dihydroxybenzoic acid forms a commensurate $(1 \times 5)$ structure, composed of deprotonated molecules. A double-row structure consisting of protonated molecules is observed for 3,5-dihydroxybenzoic acid. The positions of the functional groups steer the molecular self-assembly of dihydroxybenzoic acids in three distinct ways, namely by (a) affecting the deprotonation tendency of the acid group, (b) influencing the intermolecular interaction as already indicated by greatly different bulk structures and (c) altering the molecule-substrate matching. Our results, thus, shed light on the impact of rather small changes in the molecular structure on the structural variety in molecular self-assembly on surfaces.
\end{abstract}

www.rsc.org/pccp

On calcite, the carbonate group allows for creating a hydrogen bond to the carboxylic acid moiety of an adsorbed molecule, facilitating substrate templating. ${ }^{7}$ In the case of carboxylic acids, the charge state has to be considered: while in the protonated state hydrogen bonding steers the molecular arrangement, ${ }^{8}$ deprotonated molecules are negatively charged and can, thus, anchor towards the surface calcium cations via electrostatic attraction. ${ }^{9,10}$

Quite obviously, a common strategy to enhance the possibilities for molecule-molecule and molecule-substrate interactions is to increase the number of functional groups. ${ }^{7}$ However, not only the possible strength of a molecule-substrate interaction is important, but also the commensurability of the molecular repeat unit with the substrate periodicity has to be considered. The comparison of terephthalic acid and biphenyldicarboxylic acid on calcite has shown how the improvement of the molecule-substrate matching increases the templating effect. ${ }^{2}$

Here, we systematically study the impact of varying the positions of the hydroxyl groups at the dihydroxybenzoic acid (DHBA) core. Using non-contact atomic force microscopy (NC-AFM), we observe how the subtle differences between the isomers of DHBA result in a rich variety of structures on the clean calcite surface in ultra-high vacuum. The structures range 
from mobile molecules to strongly substrate-templated arrangements. This work illustrates that the variation of the substitution positions of the hydroxyl groups on the one hand greatly influences the chemical behavior of the molecule on the surface by changing its probability to deprotonate, which, in turn, determines the character of the molecule-substrate interaction. On the other hand, the arrangement of the functional groups influences the subtle balance of intermolecular interaction and molecule-substrate matching. The interplay of these effects determines the ability for structure formation upon molecular self-assembly demonstrating the decisive influence of the substitution position on the structural richness.

\section{Methods}

Sample preparation and NC-AFM experiments were carried out in ultra-high vacuum following a procedure reported previously. ${ }^{11}$ All NC-AFM experiments were done employing an Omicron VT-AFM at room temperature with a base pressure $<10^{-10}$ mbar. The DHBA isomers were purchased from Sigma-Aldrich (Munich, Germany) with purities as specified in Table 1. The molecules were thoroughly outgassed prior to use and sublimated in situ from a home-build Knudsen cell onto freshly cleaved calcite surfaces using the sublimation temperatures listed in Table 1.

\section{Results}

In the following, we discuss the behavior of DHBA at room temperature on the calcite (10.4) surface at sub-monolayer coverage. The calcite (10.4) surface has a rectangular unit cell of size $0.50 \times 0.81 \mathrm{~nm}^{2}$, which consists of two carbonate groups and two calcium ions (see Fig. 1a). Six constitutional isomers of DHBA exist, which differ by the positions of the hydroxyl groups at the benzene core (see Fig. 1c). Due to the different arrangement of the functional groups, the molecules differ in their chemical behavior. For example, the tendency to deprotonate is enhanced when positioning the hydroxyl groups close to the carboxyl group. This difference in the deprotonation tendency of the carboxyl group is expressed by the $\mathrm{p} K_{\mathrm{A}}$ values (see Table 1). The $\mathrm{p} K_{\mathrm{A}}$ values of the isomers reflect the distance of the hydroxyl groups to the carboxyl group. In the 2,6-DHBA molecule, both hydroxyl groups are located directly next to the carboxyl group resulting in a high deprotonation tendency $\left(\mathrm{p} K_{\mathrm{A}}=1.29\right.$ ). In the 3,4-DHBA molecule, the hydroxyl groups are located farthest away from the carboxyl group resulting in a

Table 1 Purities, sublimation temperatures $T_{\text {sub }}$ and $\mathrm{p} K_{\mathrm{A}}$ values ${ }^{12}$ of the DHBA isomers

\begin{tabular}{llll}
\hline Isomer & Purity $(\%)$ & $T_{\text {sub }}$ & $\mathrm{p} K_{\mathrm{A}}$ \\
\hline 2,6-DHBA & $>98$ & $\mathrm{RT}$ to $320 \mathrm{~K}$ & 1.29 \\
2,3-DHBA & $>99$ & $\mathrm{RT}$ & 2.94 \\
2,5-DHBA & $>99$ & $342 \mathrm{~K}$ & 2.97 \\
2,4-DHBA & $>97$ & $338 \mathrm{~K}$ & 3.29 \\
3,5-DHBA & $>97$ & $344 \mathrm{~K}$ & 4.04 \\
3,4-DHBA & $>97$ & $347 \mathrm{~K}$ & 4.48
\end{tabular}

(a)

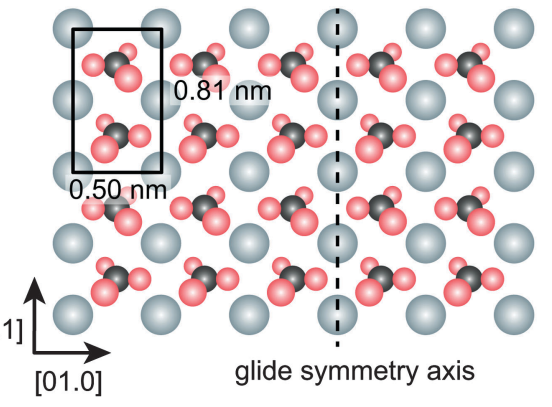

calcium

carbon

oxygen

- hydrogen

(b)

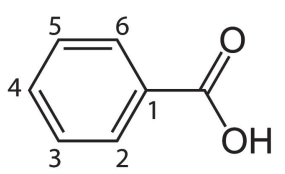

(c)

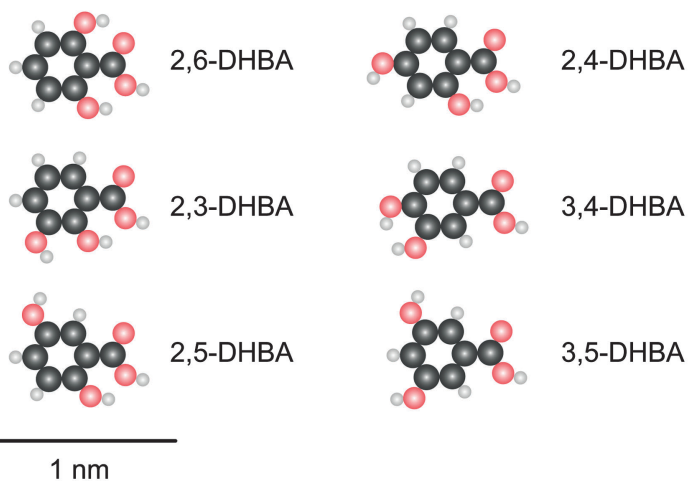

Fig. 1 Model of (a) the calcite (10.4) surface, (b) benzoic acid and (c) the constitutional isomers of dihydroxybenzoic acid (DHBA). The scale bar applies to (a) and (c).

low deprotonation tendency $\left(\mathrm{p} K_{\mathrm{A}}=4.48\right)$. In a recent study of 2,5-DHBA, we have shown that deprotonation of this isomer occurs on the calcite surface, slowly enough to be followed with the NC-AFM at room temperature. ${ }^{10}$ Spectroscopic evidence of the protonation state is difficult as most spectroscopy methods are not applicable at low molecular coverage on a bulk insulator. However, the $\mathrm{p} K_{\mathrm{A}}$ provides a reliably quantity for comparing the deprotonation tendency of carboxylic acids on the same surface. Indeed, carboxylic acids with lower $\mathrm{p} K_{\mathrm{A}}$ values than 2,5-DHBA were found to be already deprotonated on the surface, while acids with higher $\mathrm{p} K_{\mathrm{A}}$ values were found to be protonated (ref. 9 and supplementary information of ref. 9). Consequently, we expect DHBA isomers with a lower $\mathrm{p} K_{\mathrm{A}}$ value than 2,5-DHBA to tend to deprotonation as well, while DHBA isomers with a higher $\mathrm{p} K_{\mathrm{A}}$ value are expected to remain protonated.

The above consideration is a simplified picture ignoring, e.g., effects of the surface on the deprotonation process. Nevertheless, it allows for categorizing the isomers: deprotonated and protonated isomers form two groups with distinctly different molecule-substrate interactions. The interaction of protonated DHBAs with the calcite surface is likely to be dominated by hydrogen bonds between the molecules and the surface carbonate groups. ${ }^{8}$ For deprotonated molecules, on the other hand, the 
molecule-substrate interaction is expected to be governed by electrostatic interaction between the carboxylate group and the surface calcium ions. ${ }^{10}$

Intermolecular interactions between DHBA molecules decisively influence the formation of ordered patterns on the calcite surface. The bulk structure of a molecule gives a first indication of the dominant intermolecular interactions. In the bulk, all DHBAs remain protonated. Thus, the bulk structures of these molecules are dominated by hydrogen bonds. The common hydrogen bonding motif for two carboxylic acid molecules is the formation of a centrosymmetric carboxylic acid dimer. ${ }^{13}$ For benzoic acid (Fig. 1a), the bond energy of such a dimer in vacuum has been calculated to $0.74 \mathrm{eV}^{14}$ In a crystal of benzoic acid, dimers arrange into rows, which form a herringbone pattern via $\pi-\pi$ interaction. Similar arrangements are found in the bulk structures of DHBA isomers. ${ }^{15-19}$ Depending on the position of the hydroxyl groups, the dimer rows can additionally interact through hydrogen bonds. For most DHBA isomers, several polymorphs of similar energy exist, which differ in their hydrogen bond motif. The polymorphs of 3,5-DHBA do not exhibit the common arrangement into dimer rows, but show a large variety of other hydrogen bond motifs, e.g., hydrogen bonds between the carboxyl group and the hydroxyl groups. ${ }^{19}$ This finding suggests that functional groups in position 3 and 5 offer a greater variety of favorable intermolecular bond motifs besides the simple formation of carboxylic acid dimers.

When comparing isomers of the same protonation state, the strength of the molecule-substrate interaction is expected to be similar. However, the ability to form ordered structures depends crucially on the match between surface lattice and molecular arrangement which is governed by the intermolecular interactions. Consequently, the substitution positions of the functional groups are decisive for a stable substrate-templated pattern.

In the following, we will briefly describe the structural arrangement of DHBA isomers at sub-monolayer coverage on calcite (10.4). We find the individual molecules of each isomer to be mobile at room temperature on the calcite surface. Consequently, it depends on the strength and the balance of intermolecular and moleculesurface interactions whether stable structures form. In the next part of this section, we describe those molecules, which are prone to deprotonation, i.e., 2,5-DHBA, 2,3-DHBA and 2,6-DHBA. Thereafter, the arrangements of the presumably protonated molecules, namely 2,4-DHBA, 3,5-DHBA and 3,4-DHBA, are described. These descriptions will be followed by a detailed analysis in the discussion section.

\section{Structures formed by DHBA isomers with high deprotonation tendency}

The adsorption of 2,5-DHBA has been studied by our group before and two ordered phases have been identified. ${ }^{10}$ The molecules in the striped phase are protonated forming a hydrogen-bonded network, which resembles the bulk structure. Deprotonated 2,5-DHBA molecules form the densely-packed phase which consists of commensurate $(1 \times 5)$ islands. Directly after deposition, both phases are observed (Fig. 2a). Over time, the molecules in the protonated, striped islands deprotonate and the amount of striped phase decreases. The deprotonated (a)

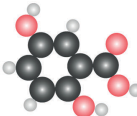

2,5-DHBA

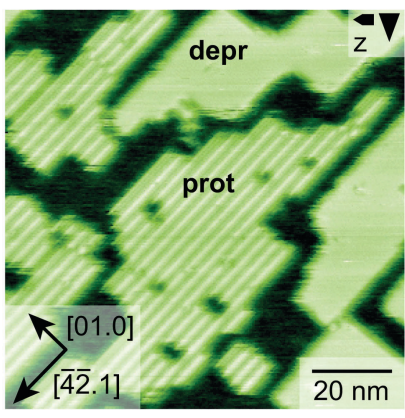

bulk-like islands deprotonate

(c)
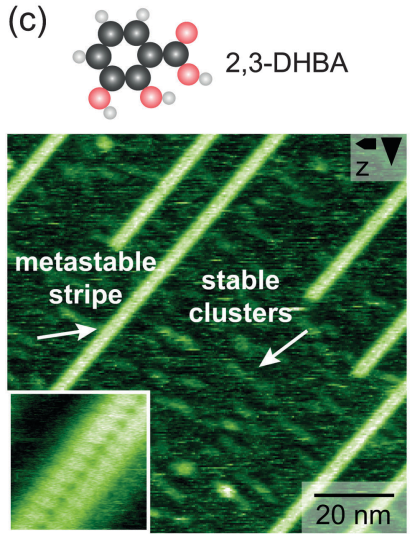

stripes transform into clusters (b)
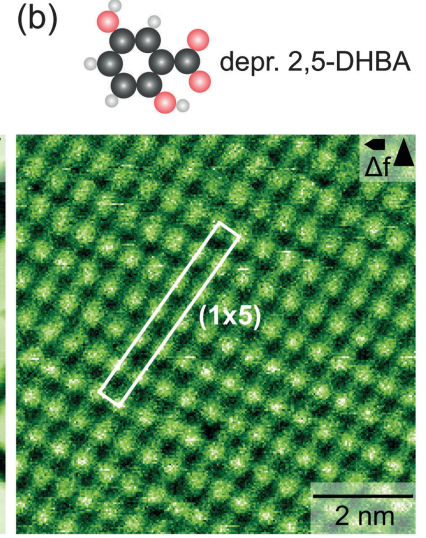

(d)
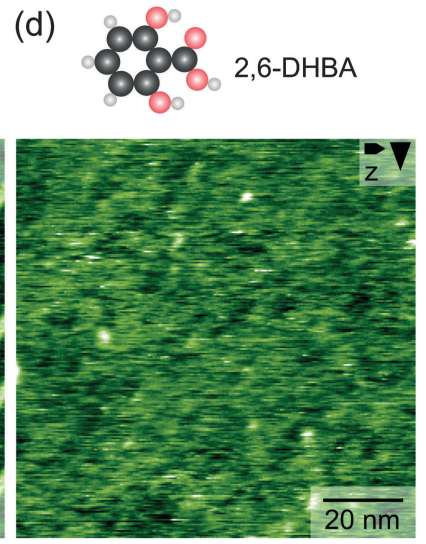

mobile
Fig. 2 Overview of all isomers prone to deprotonation after sub-monolayer deposition onto the natural cleavage plane of calcite held at room temperature. NC-AFM image of (a) 2,5-DHBA, showing both phases: striped, metastable islands that transform into densely-packed, deprotonated islands over time and (b) a dense island of deprotonated 2,5-DHBA. (c) 2,3-DHBA forms metastable stripes (see inset, $7.1 \mathrm{~nm} \times 7.1 \mathrm{~nm}$ ), which transform into stable clusters over time. (d) NC-AFM image of 2,6-DHBA revealing mobile molecules.

molecules attach to the densely-packed islands and these islands grow, indicating the mobility of the deprotonated molecules at room temperature. Eventually, only densely-packed islands in $(1 \times 5)$ arrangement remain (Fig. 2b). Thus, the previously hydrogen-bonded network formed by protonated 2,5-DHBA molecules transforms into an electrostatically stabilized structure formed by the deprotonated species.

For 2,3-DHBA, a similar phase transition is observed namely the transformation of metastable stripes into stable but unordered clusters (Fig. 2c). Directly after deposition, stripes are seen on the calcite surface which arrange along the [ $\overline{4} \overline{2} .1]$ direction. These stripes are presumably molecular double rows and exhibit a periodicity of $0.8 \mathrm{~nm}$ in [ $\overline{4} \overline{2} .1]$ direction, commensurate to the surface structure (see inset of Fig. 2c). Besides the stripes, mobile molecules and loosely arranged clusters are visible. Over time, the stripes vanish and the molecules rearrange into clusters, which are elongated along the [01.0] direction. Upon heating the sample, this phase transition is accelerated. Fig. 2(c) shows the arrangement two days after 
sublimation, exhibiting stripes as well as elongated clusters. Depending on the coverage, the complete transition from stripes to clusters happens on a time scale of several hours to many days. The $\mathrm{p} K_{\mathrm{A}}$ values of 2,5-DHBA and 2,3-DHBA are quite similar and both molecules show a phase transition. Thus, it is suggestive to ascribe the phase transitions of 2,3-DHBA to deprotonation analogous to the case of 2,5-DHBA. Consequently, we assign the metastable stripe structure formed after deposition to a hydrogen-bonded network of protonated 2,3DHBA molecules, while the stable cluster phase is assigned to the deprotonated species.

NC-AFM images of 2,6-DHBA reveal predominantly mobile molecules that are visible as streaks in the fast scan direction in Fig. 2(d). In few experiments, some additional stripe-like structures along the [01.0] direction are noted. The high tendency for deprotonation of 2,6-DHBA reflected in its low $\mathrm{p} K_{\mathrm{A}}$ value of 1.29 provides a strong indication that this molecule exists only as deprotonated species on the calcite surface. We assign the mobile species in Fig. 2(d) to deprotonated 2,6-DHBA.

(a)
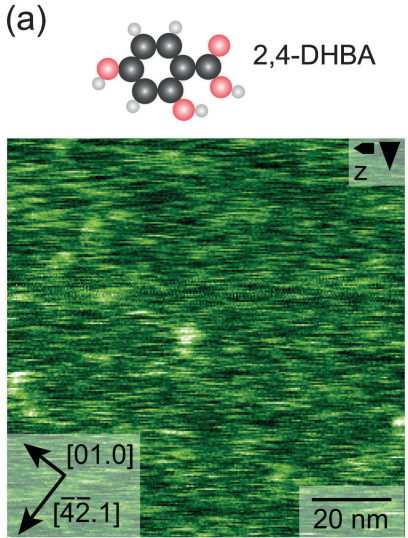

mobile

(c)
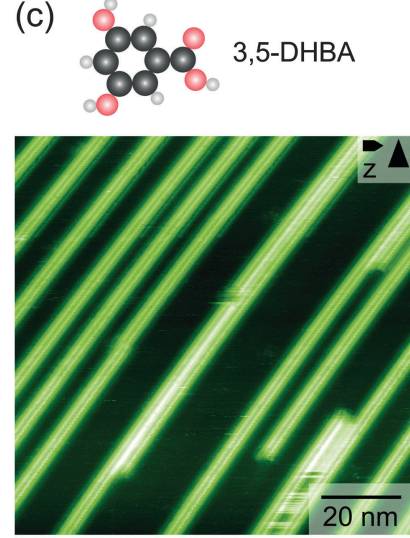

(b)
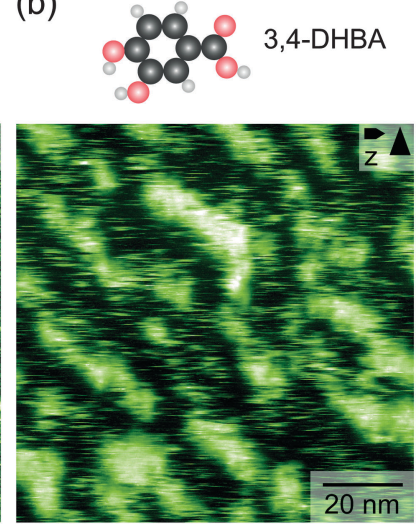

fuzzy islands

(d)

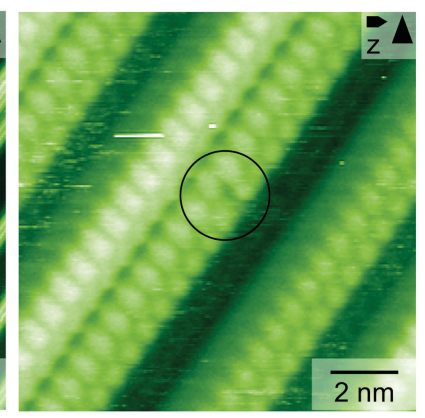

strong substrate templating

Fig. 3 Overview of all isomers expected to remain protonated after submonolayer deposition onto the natural cleavage plane of calcite held at room temperature. (a) Overview NC-AFM image of 2,4-DHBA revealing mobile molecules. (b) Overview NC-AFM image of 3,4-DHBA revealing fuzzy islands. (c) Overview NC-AFM image of 3,5-DHBA which forms stable, ordered stripes. (d) Drift-corrected, high-resolution NC-AFM image of these double stripes. A defect is visible in the center of the image.

\section{Structures formed by DHBA isomers with low deprotonation} tendency

Now we take a closer look at the DHBA isomers with a smaller deprotonation tendency as compared to 2,5-DHBA. At submonolayer coverage of 2,4-DHBA, only mobile molecules are seen in the NC-AFM images (Fig. 3a) visible as streaks in the fast scan direction. No phase transition into an ordered, substratetemplated structure is observed, even after the sample was heated to facilitate diffusion and deprotonation processes. Since no phase transition is observed and the deprotonation tendency of 2,4-DHBA is lower as compared to 2,5-DHBA we assign the mobile species in Fig. 3(a) to protonated 2,4-DHBA.

The isomer with the smallest deprotonation tendency, 3,4-DHBA, forms islands which appear elongated along the [01.0] direction (Fig. 3b). The fuzzy outline of these islands and the additional mobile species in between indicate a significant mobility of the molecule even when arranged in islands. Moreover, the islands change their shape between consecutive NC-AFM images and they are easily manipulated with the AFM tip, further indicating weak intermolecular interaction within the islands. Therefore, the internal order within an island could not be resolved. As in the case of 2,4-DHBA, no phase transition is observed even after the sample was heated. Consequently, we assign the fuzzy islands in Fig. 3(b) to be composed of protonated 3,4-DHBA.

The symmetric isomer with the two hydroxyl groups in position 3 and 5 shows a stable substrate-templated pattern

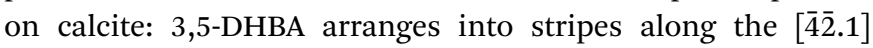
direction of the calcite surface (Fig. 3c). The stripes are typically $100 \mathrm{~nm}$ to $500 \mathrm{~nm}$ long. At low coverage, mostly individual stripes are observed. The stripes constitute molecular double rows. Drift-corrected, ${ }^{20}$ high-resolution images as shown in Fig. 3(d) reveal the molecules within the stripe are arranged in a zigzag pattern with a periodicity of $0.8 \mathrm{~nm}$ in [ $\overline{4} \overline{2} .1]$ direction. The defect marked in the center of Fig. 3(d) is caused by two missing 3,5-DHBA molecules, supporting the above-made assumption that each stripe consists of a molecular double row. The low apparent height of $0.2 \mathrm{~nm}$ and the strong directionality of the stripes, suggest a hydrogen-bonded network of flat-lying molecules. The stripes are stable even after annealing the sample to temperatures as high as $500 \mathrm{~K} .{ }^{21}$ Above this temperature the molecules start to desorb from the surface. Since no phase transition is observed and the strongly directional ordering suggest hydrogen bonds, we assign the molecules in the double rows to protonated 3,5-DHBA.

\section{Discussion}

In the following, we discuss the structural arrangements of the DHBA isomers in view of the positions of the functional groups. The preceding description of the experimental results surprisingly revealed that only two isomers of DHBA form stable, well-ordered structures at sub-monolayer coverage on the calcite surface: deprotonated 2,5-DHBA molecules form a $(1 \times 5)$ superstructure and protonated 3,5-DHBA molecules arrange into commensurate 
(a)

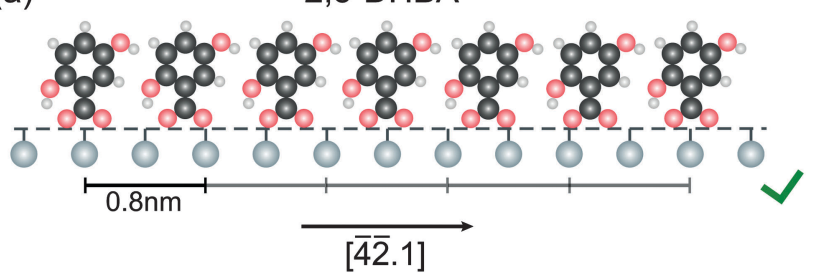

(b)

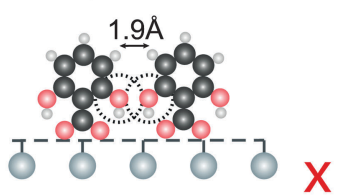

(c)

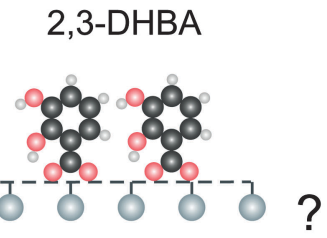

van der Waals radius

Fig. 4 (a) Model of the densely-packed phase of deprotonated 2,5-DHBA according to ref. 10. The same arrangement is prohibited for (b) 2,6-DHBA, but not impossible for (c) 2,3-DHBA.

double rows. Due to their different deprotonation state, these two isomers serve as reference in our discussion since they represent the two fundamental possibilities for moleculesubstrate interaction between DHBA and calcite. First, we compare the patterns formed by 2,6-DHBA and 2,3-DHBA with the $(1 \times 5)$ superstructure of deprotonated 2,5-DHBA, which we have proposed earlier. ${ }^{10}$ In this model, the carboxylate ions adsorb on top of the calcium cation rows along the [ $\overline{4} \overline{2} .1]$ direction due to electrostatic attraction. The comparatively small lateral repeating units do only allow for an upright-standing or tilted fashion of the molecules. Such a tilted arrangement matches the observed apparent height of $0.4 \mathrm{~nm}$. Along the same direction, neighboring 2,5-DHBA molecules align most likely via hydrogen bonds formed by the hydroxyl groups (Fig. 4a). Since the molecule-substrate interaction is dominated by the attraction between the carboxylate group and the calcium ions, it is plausible to assume that the positions of the hydroxyl groups effect the intermolecular interaction. The formation of the same ordered structure is prohibited in the case of 2,6-DHBA due to steric hindrance between the hydroxyl groups (Fig. 4b): in the configuration sketched in Fig. 4(b) the van-der-Waals radii of the neighboring hydroxyl groups $\left(1.52 \AA^{22}\right)$ overlap widely and the distance between the oxygen atoms is shorter than the bonding length of a strong hydrogen bond. Thus, it can be readily understood that deprotonated 2,6-DHBA cannot arrange into the same commensurate $(1 \times 5)$ pattern as 2,5-DHBA. For 2,3-DHBA, such a simple argument is not found (Fig. 4c). The flat clusters (apparent height smaller than $0.2 \mathrm{~nm}$ ) formed by this isomer and the mobile species in between indicate weak molecule-molecule interaction. Even though a commensurate arrangement comparable to 2,5-DHBA is not prohibited like in the case of 2,6-DHBA, the hydroxyl groups at position 2 and 3 apparently fail to stabilize an ordered structure sufficiently to prevail at room temperature. Comparing the three DHBA isomers the decisive influence of the position of each single functional group (in this

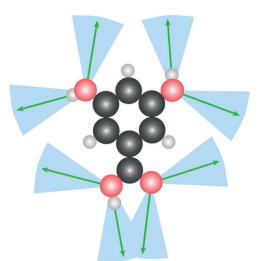

(a) 3,5-DHBA (b) 3,4-DHBA

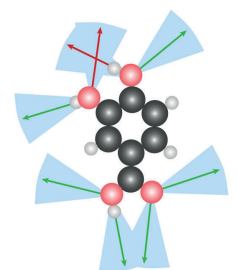

(c) 2,4-DHBA

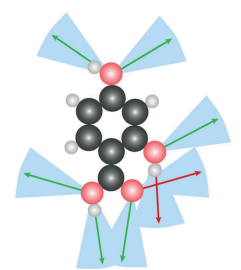

Fig. 5 Sketches of the three protonated DHBA isomers indicating all possibilities for hydrogen bonds: blue cones indicate the preferred orientation of such a bond, green arrows show hydrogen bond opportunities with other molecules or the substrate, red arrows mark hydrogen bond opportunities that block each other.

case the hydroxyl group in position 3,5 or 6 ) on the structural arrangement becomes very obvious.

In the case of protonated DHBA isomers, we expect both intermolecular and molecule-substrate interactions to be governed by hydrogen bonds. Since hydrogen bonds have a strong angular dependence, ${ }^{23}$ not only the distance of interacting partners but also the geometrical arrangement and, specifically, the angle between interacting functional groups is important for the formation of an ordered structure. The experiments show that only 3,5-DHBA forms stable, well-ordered structures, namely substrate-templated molecular double rows. Due to the vast number of possible combinations for hydrogen bonds between the multiple functional groups of the molecules and the calcite surface, identifying the correct structure for the 3,5-DHBA stripes is not trivial. With the same argument there is no naive approach that explains why 2,4-DHBA and 3,4-DHBA do not form stable, ordered structures.

However, considering the directionality of the possible hydrogen bonds we find a simple argument why the symmetric isomer is a more promising candidate for molecular selfassembly than the other two DHBA isomers. Fig. 5 shows all eight hydrogen bond opportunities for each of the three protonated isomers. Blue cones indicate the preferred direction range $\left( \pm 20^{\circ 23}\right)$ for each hydrogen bond opportunity. Obviously, (a)
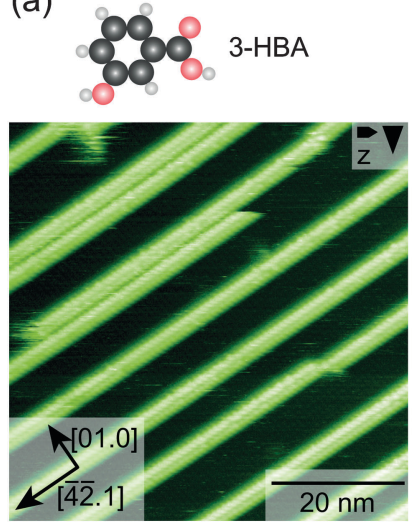

(b)
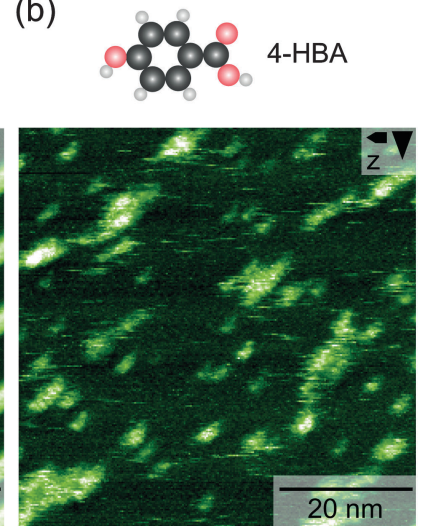

Fig. 6 NC-AFM images of (a) 3-HBA and (b) 4-HBA at sub-monolayer coverage on calcite forming stable, substrate-templated stripes and no stable structure, respectively. 
Table 2 Summary of the self-assembly of DHBA isomers on calcite

\begin{tabular}{|c|c|c|c|c|}
\hline Isomer & Observed structure & Molecule-molecule & Molecule-substrate & Assumed protonation state \\
\hline 2,3-DHBA & Molecular double-rows & \multicolumn{2}{|c|}{ Hydrogen-bonded network } & \\
\hline & Transform into clusters & \multirow{2}{*}{\multicolumn{2}{|c|}{$\begin{array}{l}\text { Electrostatics } \\
\text { Hydrogen-bonded network }\end{array}$}} & Transition from protonated to \\
\hline \multirow[t]{2}{*}{ 2,5-DHBA } & Striped islands & & & deprotonated at RT \\
\hline & Transform into dense islands & Hydrogen bonds and $\pi-\pi$ & Electrostatics & \\
\hline 3,4-DHBA & Fuzzy islands & \multicolumn{2}{|c|}{ Hydrogen-bonded network } & \\
\hline
\end{tabular}

the even arrangement of the functional groups around the benzene ring in 3,5-DHBA allows for the formation of the maximum number of hydrogen bonds towards neighboring molecules or the substrate. In the case of the two other isomers, two hydrogen bond opportunities are pairwise blocking each other. Neighboring functional groups disturb the formation of intermolecular and molecule-substrate hydrogen bonds along the directions indicated by red arrows in Fig. 5(b) and (c). Since a possible candidate for hydrogen bonding would be close to both functional groups a bond towards one of the groups is only formed if the interaction with the neighboring group is not strongly repulsive. Moreover, in the case of the hydroxyl group at position two, the formation of an intramolecular hydrogen bond with the carboxyl group is likely (Fig. 5c). Altogether, in the cases of 3,4-DHBA and 2,4-DHBA it is possible if not likely that two out of eight hydrogen bond opportunities are not accessible for intermolecular and molecule-substrate interaction and, thereby, cannot contribute to stabilize an ordered structure. This interpretation is corroborated by a comparison of 3,4-DHBA with 3-hydroxybenzoic acid (3-HBA), i.e., 3,4-DHBA without the hydroxyl group at position 4. Experiments with 3-HBA (Fig. 6a) reveal this molecule forms stable stripes on the calcite surface comparable to 3,5-DHBA. In contrast, 3,4-DHBA forms only fuzzy islands. Thus, removing one functional group (the hydroxyl in position 4) appears to restore the full potential of the remaining functional groups for forming intermolecular and molecule-substrate bonds. Further experiments show 4-hydroxybenzoic acid does not form a stable structure at sub-monolayer coverage (Fig. 6b) although the functional groups are not blocking each other in this molecule. This finding clearly demonstrates the importance of the position of each individual group within the molecule on its selfassembly properties. ${ }^{24}$

The rather different self-assembly behavior of the DHBA isomers is summarized in Table 2. Besides summarizing the observed behavior the table gives an overview about possible contributions to molecule-molecule and molecule-substrate interactions as discussed above.

\section{Conclusions}

In this work we demonstrate how a rather subtle change in the arrangement of functional groups results in a great variety of molecular structures. Dihydroxybenzoic acids on calcite (10.4) form very different structures depending on the position of the two hydroxyl groups. Among the six DHBA isomers, only 2,5-DHBA and 3,5-DHBA form stable, ordered structures on calcite at room temperature. We discuss how the positions of the hydroxyl groups steer molecular self-assembly in three distinct aspects, namely the deprotonation tendency of the DHBA molecule, the intermolecular interactions and the moleculesurface matching. Positioning the hydroxyl groups close to the carboxyl group favors deprotonation and, thereby, influences the type of molecule-substrate interaction. The distribution of the hydroxyl groups around the benzene core determines how many opportunities for intermolecular interactions are available. The specific position of each functional group is crucial for the match with the calcite surface. In summary, our study shows how positioning functional groups in a rational manner provides an option for tuning molecular self-assembly over a wide range from mobile molecules and metastable patterns to strongly substrate-templated, stable structures.

\section{Acknowledgements}

We are grateful for stimulating discussions with Wojciech Gren and Xavier Bouju. We thank the European Commission for financial support through European Project PAMS (seventh framework program GA 610446).

\section{Notes and references}

1 J.-M. Lehn, Science, 2002, 295, 2400.

2 P. Rahe, M. Kittelmann, J. L. Neff, M. Nimmrich, M. Reichling, P. Maass and A. Kühnle, Adv. Mater., 2013, 25, 3948.

3 T. Glatzel, L. Zimmerli, S. Kawai, E. Meyer, L.-A. Fendt and F. Diederich, Beilstein J. Nanotechnol., 2011, 2, 34.

4 A. Hinaut, A. Pujol, F. Chaumeton, D. Martrou, A. Gourdon and S. Gauthier, Beilstein J. Nanotechnol., 2012, 3, 221.

5 J. Schütte, R. Bechstein, M. Rohlfing, M. Reichling and A. Kühnle, Phys. Rev. B: Condens. Matter Mater. Phys, 2009, 80, 205421.

6 P. Rahe, R. Lindner, M. Kittelmann, M. Nimmrich and A. Kuhnle, Phys. Chem. Chem. Phys., 2012, 14, 6544.

7 P. Rahe, M. Nimmrich and A. Kühnle, Small, 2012, 8, 2969. 
8 D. M. Duffy and J. H. Harding, J. Mater. Chem., 2002, $12,3419$.

9 M. Kittelmann, P. Rahe, M. Nimmrich, C. M. Hauke, A. Gourdon and A. Kühnle, ACS Nano, 2011, 5, 8420.

10 M. Kittelmann, P. Rahe, A. Gourdon and A. Kühnle, ACS Nano, 2012, 6, 7406.

11 P. Rahe, J. Schütte and A. Kühnle, J. Phys.: Condens. Matter, 2012, 24, 84006.

12 Taschenbuch für Chemiker und Physiker, ed. M. D. Lechner, J. d. Ans and E. Lax, Springer, Berlin, 1992.

13 L. Leiserowitz, Acta Crystallogr., Sect. B: Struct. Crystallogr. Cryst. Chem., 1976, 32, 775.

14 D. Tzeli, I. D. Petsalakis, G. Theodorakopoulos, D. Ajami and J. Rebek, Int. J. Quantum Chem., 2013, 113, 734.

15 M. S. Adam, M. J. Gutmann, C. K. Leech, D. S. Middlemiss, A. Parkin, L. H. Thomas and C. C. Wilson, New J. Chem., 2010, 34, 85.

16 G. Giacomello, A. M. Liquori and A. Ripamonti, Nature, 1956, 177, 944.
17 L. MacGillivray and M. Zaworotko, J. Chem. Crystallogr., 1994, 24, 703-705.

18 A. Parkin, M. Adam, R. I. Cooper, D. S. Middlemiss and C. C. Wilson, Acta Crystallogr., Sect. B: Struct. Sci., 2007, 63, 303.

19 B. Sarma, P. Sanphui and A. Nangia, Cryst. Growth Des., 2010, 10, 2388.

20 P. Rahe, R. Bechstein and A. Kühnle, J. Vac. Sci. Technol., B, 2010, 28, C4E31-C4E38.

21 Temperatures are not measured directly at the sample but close to the heating stage. The temperature of the sample during annealing is estimated using a calibration curve provided by the AFM manufacturer.

22 CRC Handbook of Chemistry and Physics, ed. D. R. Lide, CRC Press/Taylor and Francis, Boca Raton, FL, 89th edn (Internet Version 2009), 2009.

23 T. Steiner, Angew. Chem., Int. Ed., 2002, 41, 48.

24 We expect 3-hydroxybenzoic acid and 4-hydroxybenzoic acid to be protonated on the calcite surface, since their $\mathrm{p} K_{\mathrm{A}}$ values (ref. 21) are 4.08 and 4.57 , respectively. 